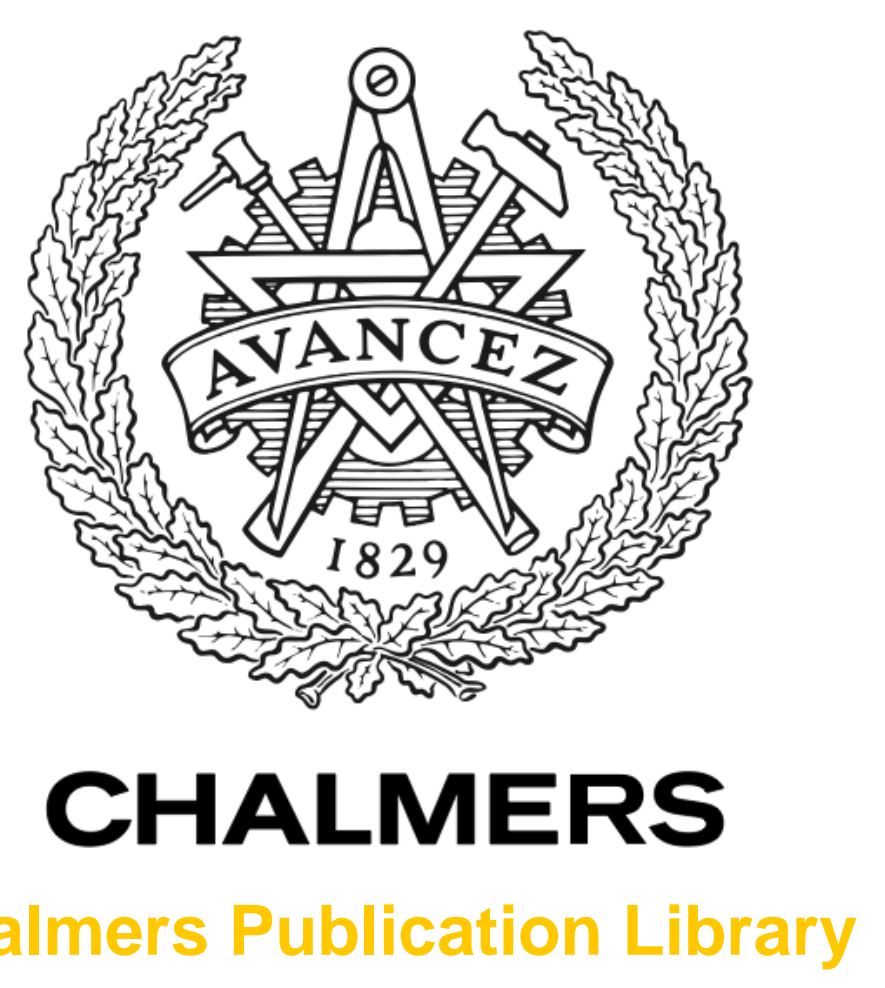

Chalmers Publication Library

\title{
Evolution of an UWB antenna for hyperthermia array applicator
}

This document has been downloaded from Chalmers Publication Library (CPL). It is the author's version of a work that was accepted for publication in:

Proceedings of 6th European Conference on Antennas and Propagation, EuCAP 2012. Prague, 26-30 March 2012.

Citation for the published paper:

Dobsicek Trefna, H. ; Imtiaz, A. ; Lui, H. (2012) "Evolution of an UWB antenna for hyperthermia array applicator". Proceedings of 6th European Conference on Antennas and Propagation, EuCAP 2012. Prague, 26-30 March 2012. pp. 1046 - 1048.

http://dx.doi.org/10.1109/EuCAP.2012.6206294

Downloaded from: http://publications.lib.chalmers.se/publication/168795

Notice: Changes introduced as a result of publishing processes such as copy-editing and formatting may not be reflected in this document. For a definitive version of this work, please refer to the published source. Please note that access to the published version might require a subscription.

Chalmers Publication Library (CPL) offers the possibility of retrieving research publications produced at Chalmers University of Technology. It covers all types of publications: articles, dissertations, licentiate theses, masters theses, conference papers, reports etc. Since 2006 it is the official tool for Chalmers official publication statistics. To ensure that Chalmers research results are disseminated as widely as possible, an Open Access Policy has been adopted.

The CPL service is administrated and maintained by Chalmers Library. 


\title{
Evolution of an UWB Antenna for Hyperthermia Array Applicator
}

\author{
Hana Dobšíček Trefná*, Azeem Imtiaz ${ }^{\dagger}$, Hoi-Shun Lui*, Tonny Rubæk* and Mikael Persson* \\ *Dept. Signal and Systems, Chalmers University of Technology, \\ Hörsalsvägen 11, Gothenburg, Sweden \\ Email: hanatre@chalmers.se \\ ${ }^{\dagger}$ Cardiff School of Engineering, Cardiff University, \\ Newport Road,Cardiff,Wales,UK
}

\begin{abstract}
Designs of three UWB antennas and their suitability to be used as a basis antenna element in the hyperthermia applicator for the treatment of tumors in head and neck region are considered. The antennas with reflection coefficient less than - $10 \mathrm{~dB}$ at frequency range $350 \mathrm{MHz}$ to $1 \mathrm{GHz}$ are immersed in water bolus. The evaluation of the SAR distributions was carried out by comparing the different cross-sections of muscle equivalent phantom. Numerical simulations were performed in CST Microwave studio and verified experimentally via measurements of S-parameters and SAR distributions in phantoms.
\end{abstract}

\section{INTRODUCTION}

Hyperthermia is presently used as an adjuvant to the radiation therapy in the treatment of certain types of cancers. Recently, randomised trials have shown a significant advantage of combining hyperthermia with radiotherapy and/or chemotherapy in the treatment of solid tumours [1]-[3]. The objective of hyperthermia treatment is to raise the temperature in the tumour to a therapeutic level $41^{\circ} \mathrm{C}-44^{\circ} \mathrm{C}$ for a sufficient period of time to achieve cell death or render the cells more sensitive to ionizing radiation and chemical toxins. The present challenge is adequately heating of deep seated tumours while preventing surrounding healthy tissue from undesired overheating and damage.

In order to improve the heat delivery to the tumor, we are developing a flexible applicator, which is tumor-volumespecific [4], [5]. In other words, an applicator that is capable of modifying the focus size depending of the tumour position and volume. The importance of the foci-spot size adjustments comes from the ability to restrain hot spots near the tumour, which are difficult to suppress. An adaptation of the heating pattern can be realised by varying the operating frequency of the antennas and potentially by the use of UWB pulse sequences instead of pure harmonic signals, as used in the present heating equipment.

In this contribution, we present a design of three UWB antennas and their suitability to be used as a basis antenna element in the hyperthermia applicator for the treatment of tumors in Head \& Neck region. Since the optimal frequency for a specific treatment is dependent on the tumour positioning and size, our system is designed for a wideband frequency range of $350 \mathrm{MHz}$ to $1 \mathrm{GHz}$.
The first antenna design is triangular patch antenna [5] used presently in our hyperthermia system prototype. This antenna however does not cover the whole frequency band and demands matching liquid change in water bolus to enable employment of specific treatment. In order to enable full utilisation of a UWB regime, a stacked $\pi$-slot antenna was developed. Although this antenna covers the whole frequency band, it is not optimal in intended operation due to the unfavourable E-field pattern at higher frequencies. Therefore we desided to further investigate a general suitability of the balanced antipodal Vivaldi antenna of Bourqui et. al. [7] for hyperthermia application.

\section{Single ANTENNA DESIGN}

During hyperthermia treatment, a water bolus filled with demineralised water is typically placed between the body and the applicator. The water bolus reduces hot spots and improves impedance matching between the biological tissue and the antennas. In the antenna designs proposed in this study, the antennas are immersed in the water bolus $(\epsilon=78, \sigma=0.1$ $\mathrm{S} / \mathrm{m}$ ), which considerably decreases the size of the antenna. All antennas are fed by coaxial line.

\section{Triangular patch antenna}

The triangular shape was selected for its similar radiation properties to rectangular patches. It have the added advantage of being physically smaller. The dimensions of the antennas were further reduced by using a shorting wall connected to the edge of the patch and the ground plan. The broadband behaviour obtained is the result of the currents along the edges of the slots, which introduces additional resonances in conjunction with the resonance of the main patch. The slots also introduces capacitive reactance that counteracts the inductive reactance of the feed.

The permittivity of the bolus affects the matching of the antenna. It is thus possible to tune the applicator to different frequencies by changing the permittivity from treatment to treatment.

\section{Stacked $\pi$-slot antenna}

The wideband nature of the antenna is realized by adding coplanar $\pi$-shaped parasitic elements around the radiating 

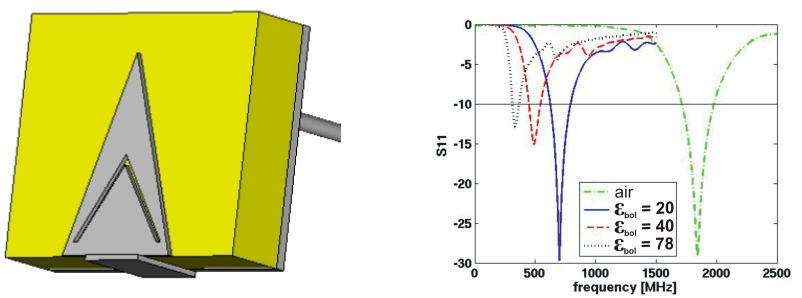

Fig. 1. (a) Geometry of the triangular patch antenna. (b) Return loss.

edges of the fed patch and stacking a rectangular patch above them. The lower patch antenna is directly fed by either coaxial cable while upper patches are parasitically fed by the coupling with lower patch antenna. In order to decrease the size of the antenna, two shorting pins are used. The antenna is fed by coaxial cable which introduces an inductance into the feed. In order to compensate for it, a metallic circular washer is placed between ground plane and lower patch. The overall antenna size is $80 \times 65 \times 19.65 \mathrm{~mm}$.

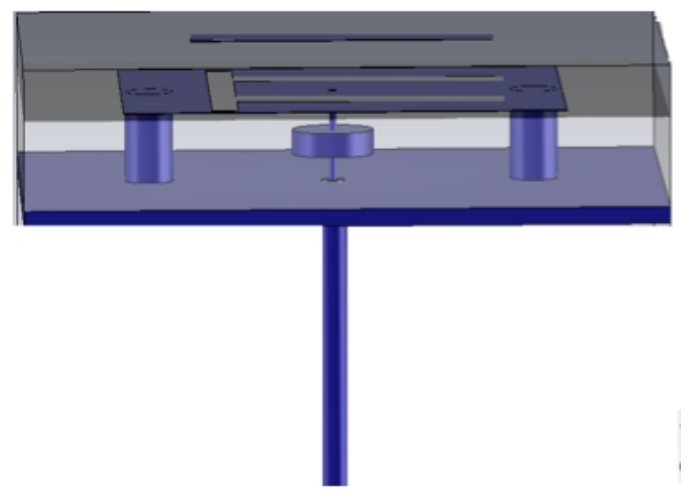

Fig. 2. Geometry of the stacked $\pi$-slot antenna.

\section{Vivaldi antenna}

The Vivaldi antenna consists of three copper layers; the two external layers are the ground planes and the central layer is the conductor. The copper layers are separated by two dielectric substrates and two additional dielectric layers are stacked on each side of the antenna, which balances the dielectric loading between the conductor and ground planes. As a result, the usual beam squint observed in this type of antenna construction is reduced [6]. In this study used a scaled version of the balanced antipodal Vivaldi antenna of Bourqui et. al. [7]. The overall antenna dimensions are 111x66x13.8 mm excluding SMA connector.

\section{RESUlts}

A muscle tissue load configuration, consisting of a single antenna element and a 12-cm-thick slab of homogeneous muscle tissue phantom $(\epsilon=56, \sigma=0.8 \mathrm{~S} / \mathrm{m})$. The distance between the phantom and the stacked patch antenna was initially $2.45 \mathrm{~cm}$, while in the case of Vivaldi antenna the distance was set to $40 \mathrm{~cm}$.

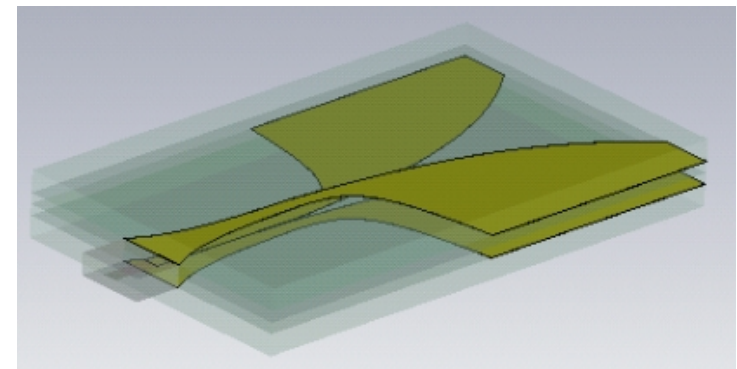

Fig. 3. (a) Geometry of the Vivaldi antenna.

\section{Stacked $\pi$-slot antenna}

Figure 4 shows the return loss of the $\pi$-stacked patch antenna. The operation frequency band of the antenna ranges from 390 to $1090 \mathrm{MHz}$. There is, however, a slight mismatch occuring at frequencies between 600 and $750 \mathrm{MHz}$. This is caused by substitution of the ideal parameters in the original design by the standardised values.

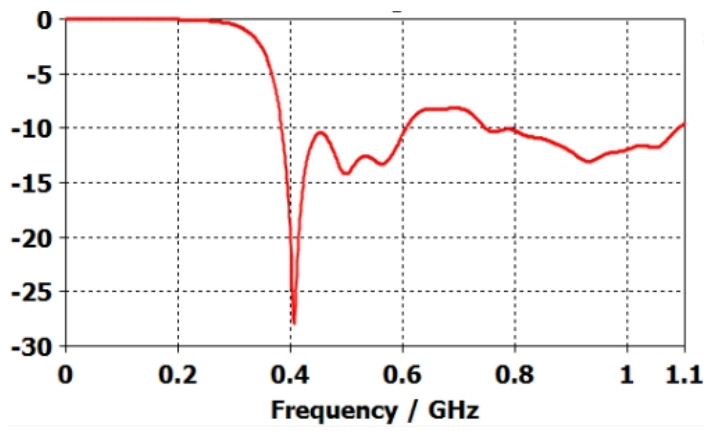

Fig. 4. Return loss of the stacked $\pi$-slot antenna.

The SAR distributions in the muscle phantom for frequencies of 434, 800 and $1000 \mathrm{MHz}$ are shown in Figure 5. In the low-frequency case (frequencies up to $800 \mathrm{MHz}$ ), the SAR distribution is fairly homogeneous over the size of the antenna. Above $850 \mathrm{MHz}$, the SAR distribution is becoming non-uniform with two dominant side lobes. Since the $\pi$-slot does not longer compensate for the inductance of the shorting pins, they start to radiate. A potential solution to this problem could be the removal of the shorting pins and decrease of the washer radius, nevertheless the operational frequency band of the antenna will substantially reduce.

\section{Vivaldi antenna}

Figure 6 shows the return loss of the Vivaldi antenna. Reflection coefficient is below $-10 \mathrm{~dB}$ above $360 \mathrm{MHz}$ and up to $5 \mathrm{GHz}$, no upper limit has been found.

The SAR distributions of Vivaldi antenna in the muscle phantom for frequencies of 434, 800 and $1000 \mathrm{MHz}$ are shown in Figure 7. The SAR distribution is homogeneous over the whole frequency band.

It is worth mentioning that effieciency of the Vivaldi antenna is significantly lower than patch antenna. It can be seen from comparison of scales in Figures 5 and 7 that SAR values are 


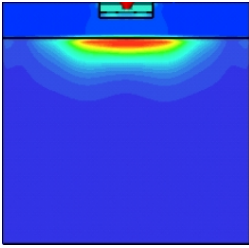

(a)

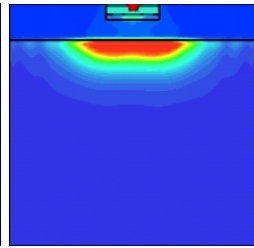

(b)

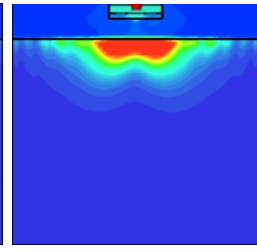

(c)
Fig. 5. Simulated SAR distribution of the $\pi$-stacked patch antenna in muscle phantom for different frequencies on the Y plane @ ( $y=0)$. (a) $f=434 \mathrm{MHz}$. (b) $\mathrm{f}=800 \mathrm{MHz} \cdot \mathrm{f}=1 \mathrm{GHz}$

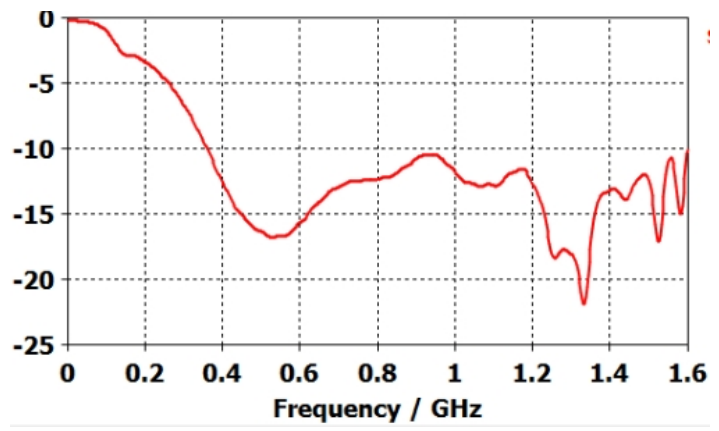

Fig. 6. (a) Return loss of the Vivaldi antenna.

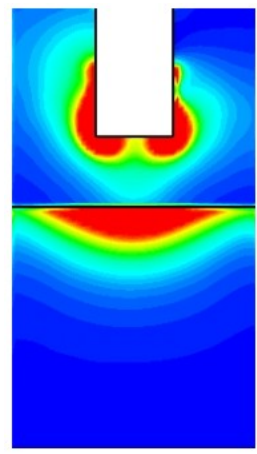

(a)

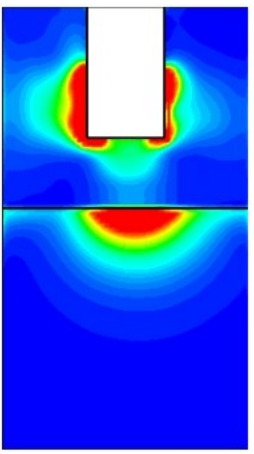

(b)

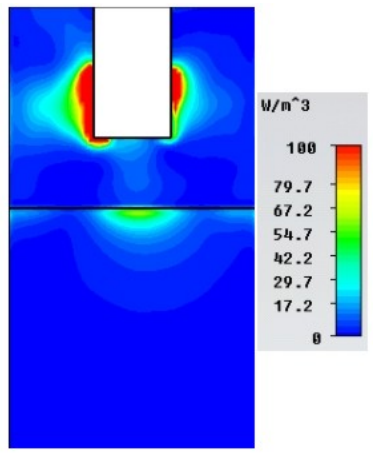

(c)
Fig. 7. Simulated SAR distribution of the Vivaldi antenna in muscle phantom for different frequencies on the Y plane @ ( $\mathrm{y}=0$ ). (a) $\mathrm{f}=434 \mathrm{MHz}$. (b) $\mathrm{f}=800 \mathrm{MHz} . \mathrm{f}=1 \mathrm{GHz}$

at least 10 times higher in patch antenna case. The radiation characteristics of the Vivaldi antenna can be, for the costs of simple structure, improved by using of "director" as suggested by Bourqui et.al. [8].

\section{CONCLUSION}

Triangular patch antenna does not cover the whole frequency band and demands matching liquid change in water bolus to enable employment of specific treatment. While clinically possible, this is highly impractical and hinder full utilisation of a UWB regime. The bandwidth requirements can be resolved by the stacked $\pi$-slot patch antenna. There are nevertheless other issues related with the design, such as unfavourable E-field pattern at higher frequencies, complicated structure and increasing price, which hamper using of this antenna in intended operation. Although the lower efficiency, the Vivaldi antenna seems to be the most appropriate design for our application. It fully covers the desired frequency bandwitdh, while being of simple structure and less expensive than the stacked patch antenna.

\section{REFERENCES}

[1] J. Van der Zee, D. Gonzáles Gonzáles, et al.," Comparison of radiotherapy alone with radiotherapy plus hyperthermia in locally advanced pelvic tumours: a prospective, randomised, multicentre trial", Lancet, vol. 355 , pp. 1119-1125, 2000

[2] E.L. Jones, J.R. Oleson, et al.,"Randomized Trial of Hyperthermia and Radiation for Superficial Tumors", J Clin Oncol., vol. 23, pp. 30793085,2005

[3] R. Colombo, et al.,"Multicentric Study Comparing Intravesical Chemotherapy Alone and With Local Microwave Hyperthermia for Prophylaxis of Recurrence of Superficial Transitional Cell Carcinoma.", J Clin Oncol., vol. 21, pp. 4270-4276, 2003.

[4] H. Dobšíček Trefná, J. Vrba, and M. Persson, "Time-Reversal Focusing in Microwave Hyperthermia for Deep-Seated Tumours", Phys Med Biol, vol 55, pp. 2167-85, 2010.

[5] H. Dobšíček Trefná, J. Vrba, M. Persson, ”Evaluation of a patch antenna applicator for time reversal hyperthermia", Int J Hyperthermia, vol. 26, pp. 185-97, 2010.

[6] J. Langley, P. Hall, and P. Newham, "Novel ultrawide-bandwidth Vivaldi antenna with low crosspolarisation," Electron. Lett., vol. 29, pp. 20042005, 1993.

[7] J. Bourqui, M. Okoniewski, E.C. Fear,'Balanced Antipodal Vivaldi Antenna for breast cancer detection," In Proc. 2nd Eur. Conf. Antennas Propag., Edinburgh, U.K.,2007.

[8] J. Bourqui, M. Okoniewski, E.C. Fear,'Balanced Antipodal Vivaldi Antenna With Dielectric Director for Near-Field Microwave Imaging,'IEEE Trans.Ant. Propag., vol. 58, pp.2318 - 2326, 2010. 